\title{
SOBRE EL TEOREMA DE HAHN-BANACH EN ESPA CIOS LOCALMENTE K-CONVEXOS
}

\section{JAIME SEGUEL CANPODONICO}

\section{CUERPOS ESFERICO - COMPLETOS.}

Sea $K$ un cuerpo, $K$ se dice no - arquimediano si $\psi: K \rightarrow R$ tal que :

$$
\begin{array}{lll}
(\mathrm{V} 1) & \psi & (\alpha)>0 \\
(\mathrm{~V} 2) & \psi & (\alpha)=0 \\
(\mathrm{~V} 3) & \psi & (\alpha \beta)=>\alpha=0 \\
(\mathrm{~V} 4) & \psi & (\alpha+\beta)<(\alpha) \psi(\beta)
\end{array}
$$

Anotamos $\psi(\alpha)=|\alpha|$ y la llamamos valuación no arquimediana sobre $K$.

Nótese que (V1), (V2) y (V3) son propiedades del módulo complejo. De ellas (tal como en el caso complejo) se desprenden :
(1)$$
\left|\begin{array}{r}
1 \\
-1 \\
-\alpha
\end{array}\right|
$$$$
=1
$$
$=1$$$
-|\alpha|
$$
Además de (V4) podemos implicar: $|\alpha+\beta|<|\alpha|+|\beta|$ - sea, $\mid$ | es una norma sobre $k$. 
Tenemos además la siguiente propiedad:

( 5 )

$$
\begin{array}{lrl}
|\alpha|<|\beta| \rightarrow|\alpha+\beta| & =|\beta| \\
\text { En efecto: de (V4) : }|\alpha+\beta| & \leqslant|\beta| \\
\text { si suponemos } & |\alpha+\beta|<|\beta| \\
\text { entonces: } & |\beta|=|\alpha+\beta-\alpha| \\
& <\operatorname{máx}\{|\alpha+\beta||-\alpha|\}
\end{array}
$$

o sea : $|\beta|<\mid \alpha$ a $\beta|| \beta|<| \alpha \mid$

lo que es contradictorio. \#

$(k,||)$ es un espacio topológico y las vecindades basales de $\alpha \circ \varepsilon k$ son los discos abiertos:

$$
\stackrel{\circ}{B}_{r}(\alpha \circ)=\{\alpha \varepsilon K /|\alpha-\alpha \circ|<r\}
$$

discos.

$$
\text { Los discos cerrados serán llamados, brevemente: }
$$

$$
B_{r}(\alpha \circ)=\{\alpha \in K /|\alpha-\alpha \circ| \leqslant r\}
$$

es el disco cerrado de centro $\alpha \circ$ y radio $r$ :

PROPOSICION 1 :

$$
\begin{aligned}
& B_{r 1}\left(\alpha_{1}\right) \cap B_{r 2}\left(\alpha_{2}\right) \neq \varnothing \Lambda r_{1} \leqslant r_{2} \\
& B_{r 1}\left(\alpha_{1}\right) \subset B_{r 2}\left(\alpha_{2}\right)
\end{aligned}
$$

$\underline{\text { DM }}:$

$$
\operatorname{sean}:\left\{\begin{array}{llll}
\alpha^{*} & \varepsilon & B_{r 1} & \left(\alpha_{1}\right) \cap B_{r 2}\left(\alpha_{2}\right) \\
\alpha & \varepsilon & B_{r 1} & \left(\alpha_{1}\right)
\end{array}\right.
$$




$$
\text { entonces : } \begin{aligned}
\left|\alpha-\alpha_{2}\right| & =\mid\left(\alpha-\alpha_{1} j+\left(\alpha_{1}-\alpha^{*}\right)+\left(\alpha * \alpha_{2}\right) \mid\right. \\
\leqslant & \max \left\{\left|\alpha-\alpha_{1}\right|,\left|\alpha_{1}-\alpha *,\right| \alpha^{*}\left|\alpha_{2}\right|\right\} \\
< & r_{2}
\end{aligned}
$$

Se sigue de esta proposición que : Dada una familia finita de discos tal que dos cualesquiera de sus ele mentos tienen intersección no vacia, entonces la intersec ción sobre toda la familia es no vacia. Realmente, ella pue de ordenarse totalmente por la inclusion.

Es claro, también, que si $\beta$ es una familia arbitraria de discos tal que dos cualesquiera de sus miembros, tienen intersección no vacia, entonces, ( $\beta$, ) es totalmente ordenado; sin embargo,

$$
\bigcap_{\beta} \beta
$$

puede ser vacia a menos que K satisfaga un cierto requisito de completitud. Esta observación motiva la siguiente defini
ciôn :

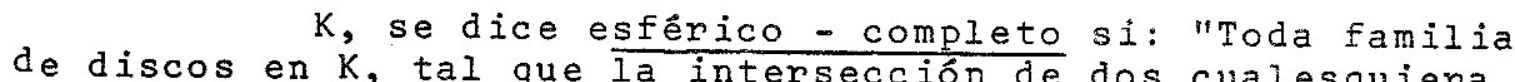
de sus miembros es no vacia, tiene interseccion cualesquiera

PROPOSICION 2 :

$$
K \text { esfêrico - completo } \Rightarrow K|| \mid \text { - completo. }
$$

DM :

$$
\begin{aligned}
& \text { sean: } \begin{cases}\left(\alpha_{n}\right) & \text { sucesión } \mid- \text { - cauchy en } k \\
B_{n}= & \left\{\alpha \varepsilon k /\left|\alpha-\alpha_{n}\right| \leqslant \max \left|\alpha_{k}-\alpha_{k+1}\right|\right\}\end{cases} \\
& k \geqslant n
\end{aligned}
$$

entonces : $\alpha_{n+1} \quad \varepsilon B_{n} \cap B_{n+1}$

además: $\max \left|\alpha_{k}-\alpha_{k+1}\right| \geqslant \max \left|\alpha_{k}-\alpha_{k+1}\right|$

$k \geqslant n \quad k \geqslant n+1$ 
de donde: $B_{n} \supset B_{n+1}, \forall n$.

En consecuencia : $B_{n} \cap B_{m} \neq \emptyset, \forall n, m$.

Luego, como $\mathrm{K}$ es esférico-completo: $\bigcap_{\mathrm{n} \varepsilon \mathrm{N}} \mathrm{B}_{\mathrm{n}} \neq \emptyset$

sea $\alpha_{0} \varepsilon \bigcap_{n \varepsilon N} B_{n}$

Obviamente: $\alpha_{n} \rightarrow \alpha_{0}$ en $\mid 1$.

\#

Examinamos a continuación la clase de sucesiones que va ligada a esta noción de completitud esférica. Una sucesión $\left(\alpha_{n}\right)$ en $K$ se dice pseudo convergente. si :

$$
\mathrm{n}<\mathrm{m}<\mathrm{s} \Rightarrow\left|\alpha_{s}-\alpha_{m}\right|<\left|\alpha_{m}-\alpha_{n}\right|
$$

PROPOSICION 3 :

Si $\left(\alpha_{n}\right)$ es pseudo convergente; entonces:

$\mathrm{n}<\mathrm{m} \Rightarrow\left|\alpha_{\mathrm{m}}-\alpha_{\mathrm{n}}\right|=\left|\alpha_{\mathrm{n}+1}-\alpha_{\mathrm{n}}\right|$

$\underline{D M}:$

$$
\begin{aligned}
& n<n+1 \leqslant m \Rightarrow\left|\alpha_{m}-\alpha_{n+1}\right|<\left|\alpha_{n+1}-\alpha_{n}\right| \\
& \text { pero: }\left|\alpha_{m}-\alpha_{n}\right|=\left|\left(\alpha_{m}-\alpha_{n+1}\right)+\left(\alpha_{n+1}-\alpha_{n}\right)\right| \\
& \left|\alpha_{n+1}-\alpha_{n}\right|
\end{aligned}
$$

esta ulltima igualdad gracias a (5). 
Se llama pseudo-limite de la sucesión pseudo-con
vergente $\left(\alpha_{n}\right)$, a un elemento $\alpha_{0} \varepsilon k$ tal que :

$$
\left|\alpha_{0}-\alpha_{n}\right|=\left|\alpha_{n+1}-\alpha_{n}\right|, \forall n \in N
$$

TEOREMA :

Para que $K$ sea esférico - completo es necesa rio y suficiente que toda sucesión pseudo - convergente eñ $\mathrm{k}$, tenga un pseudo - limite.

$\underline{\mathrm{DM}}:$

Suponiendo $K$ esfêrico - completo y $\left(\alpha_{n}\right)$ pseudo - con vergente en $K$.

$$
\begin{aligned}
& \text { sea : }{ }^{B}=\left\{\alpha \cdot k /\left|\alpha-\alpha_{n}\right| \leqslant\left|\alpha_{n+1}-\alpha_{n}\right|\right\} \\
& \text { sean: } \mathrm{m}, \mathrm{n} \varepsilon \mathrm{N} \\
& \text { Luego: } \alpha_{\mathrm{m}} \quad \varepsilon \mathrm{B}_{\mathrm{n}} \\
& \text { o sea : } B_{m} \cap B_{n} \neq \varnothing \\
& \mathrm{n} E \cdot \mathrm{N} \\
& \text { sea : } \alpha_{\circ} \varepsilon \cap B_{n}
\end{aligned}
$$$$
\text { Si }: n<m,\left|\alpha_{m}-\alpha_{n}\right|=\left|\alpha_{n+1}-\alpha_{n}\right|
$$

Supongamos: $n_{0} \varepsilon N$ tal que $\left|\alpha_{0}-\alpha_{n_{0}}\right|<\left|\alpha_{n_{0}+1}-\alpha_{n_{0}}\right|$

$$
\text { Entonces : }\left|\alpha_{0}-\alpha_{n_{0}}+1\right|=\left|\left(\alpha_{0}-\alpha_{n_{0}}\right)+\left(\alpha_{n_{0}}-\alpha_{n_{0}+1}\right)\right|
$$




$$
=\left|\alpha_{n_{0}}-\alpha_{n_{0}}+1\right| \text { (gracias a (5). }
$$

Ahora, de :

$$
\begin{aligned}
& \mathrm{n}_{0}<\mathrm{n}_{0}+1<\mathrm{n}_{0}+2<\mathrm{n}_{0}+3 \\
& \text { se tiene: }\left|\alpha_{0}-\alpha_{n_{0}+2}\right|=\left|\left(\alpha_{0}-\alpha_{n_{0}+1}\right)+\left(\alpha_{n_{0}+1}-\alpha_{n_{0}+2}\right)\right| \\
& =\left|\left(\alpha_{n_{0}}-\alpha_{n_{0}+1}\right)+\left(\alpha_{n_{0}+1}-\alpha_{n_{0}+2}\right)\right|\left|\alpha_{n_{0}}-\alpha_{n_{0}+1}\right|> \\
& \left|a_{n_{0}}+2-\alpha_{n}+3\right| \\
& \text { o sea : } \alpha_{0} \quad \notin \mathrm{B}_{\mathrm{n}_{0}+2} ; 10 \text { que es contradic }
\end{aligned}
$$

torio.

Suponiendo que toda sucesión pseudo-convergente admite un pseudo-limite, sea 8 una familia de discos tal que dos cualesquiera de sus miembros tienen intersección no vacia; entonces $(B, C)$ es totalmente ordenado.

$$
\begin{aligned}
& \text { sea } r_{B} \text { el radio de } B \varepsilon B \\
& \text { y sea }: r=\underset{B \in \&}{i n f} \quad r_{B}
\end{aligned}
$$

Entonces :
a) $\int \exists B_{0} \varepsilon \beta$ tal que $r=r_{0}$
b) $\exists\left(B_{n}\right)<\beta$ tal que $r_{n}^{+} r$.

Si a) $\quad B_{0}=\bigcap_{B \varepsilon \beta}^{B} \neq \varnothing$ 
si. b) J $\left(a_{n}\right)$ sucesión tal que : $\alpha_{n} \varepsilon B_{n}$ y $\alpha_{n} \not B_{n+1}$

si $n<m<s$, entonces:

$\alpha_{s} \quad \varepsilon \quad B_{s}<B_{m}$ y $\alpha_{m} \quad \varepsilon \quad B_{m}$

luego :

$\left|\alpha_{m}-\alpha_{s}\right| \leqslant r_{B}$

Ahora : $\alpha_{\mathrm{n}} \notin \mathrm{B}_{\mathrm{m}}$

o sea : $\left|\alpha_{\mathrm{m}}-\alpha_{\mathrm{n}}\right|>\mathrm{r}_{\mathrm{B}}$

de donde : $\left|\alpha_{s}-\alpha_{m}\right|<\left|\alpha_{n}-\alpha_{m}\right|$

$\therefore \quad\left(\alpha_{n}\right)$ es pseudo-convergente

sea $\alpha_{0}$ su pseudo limite.

luego: $\left|\alpha_{0}-\alpha_{n}\right|=\left|\alpha_{n+1}-\alpha_{n}\right| \leqslant r_{n} \quad \forall n$

Por 10 tanto: $\alpha_{0} \varepsilon B_{n}, \quad \forall n$

de donde :

$\alpha_{\circ} \varepsilon \bigcap_{B \in B} B$

II. ESPACIOS LOCALMENTE $K$ - CONVEXOS.

Un espacio vectorial topológico es un espacio vec torial dotado de una cierta topologia $\tau$ que hace continuas las operaciones de adición vectorial y producto de escalar por vector; $\tau$ se dice, en ese caso, compatible con la estructura lineal de E. 
Una clara consecuencia de la continuidad de las operaciones antes mencionadas es el par de hechos siguientes: dad de $x \in E$.

(A) Si U es vecindad de 0 en $E, x+U$ es vecin

(B) Si U es vecindad de 0 en $E, \lambda U$ es vecindad de $0, \lambda$ escalar.

Un modo de definir estas topologias es a partir de la base del filtro de vecindades de 0 , o del filtro de ve cindades de 0 .

Al respecto, en teoría de espacios vectoriales topológicos, se tiene el resultado fundamental siguiente:

"Un filtro $\widetilde{Z}$ en un espacio vectorial E, es el filtro de vecindades de 0 de un topologia compatible con la estructura algebraica (o linea..) de E si y solo si:
1) $0 \quad \varepsilon \quad U$,
$\forall U \quad \varepsilon \widetilde{c}$
2) $\forall \mathrm{U}$
$\varepsilon \mp$,
习 $\mathrm{V} \varepsilon$ 元
tal que: $V+V$
3) $\forall \mathrm{U}$
$\varepsilon \mathcal{E}$,
4) $\forall \mathrm{U}$
$\varepsilon \widetilde{\tau}$, $\forall$
$\lambda$ escalar, $\lambda \neq 0, \lambda U \varepsilon F$
5) $\forall U$
$\varepsilon \widetilde{\tau}$.
$\exists \vee c i$
$U$ es absorbente

La obsorción y el equilibrio son dos buenas propiedades geométricas, indispensables para asegurar la continuidad del producto externo.

Un filtro $\mathcal{E}$ que satisface además: 6) $\forall U \in T, \exists V \subset L^{\prime}, \quad V$ convexo
se llama convexo.

Un espacio vectorial topológico dotado de una to pologia definida por un filtro que satisface de (1) a (6), es llamado localmente convexo. sultado siguiente :

Para espacios localmente convexos se tiene el re

"La topología de un espacio localmente convexo puede definirse en base a una familia de semi-normas $\{\mathrm{p} \lambda /$ $\lambda \varepsilon \Gamma\}$; entendiendo por tales aplicaciones subaditivas y homogéneas de $E$ en $R "$.

Para el caso en que $K$ es no-arquimediano estable 
cemos el resultado análogo. Partiendo de la noción de $k$ con vexo.

Sea E un espacio vectorial sobre un cuerpo no-ar quimediano $K$. ; $S<E$ se dice $K$-convexo si :

$\forall x, y \varepsilon S, \forall \lambda, \mu \varepsilon K$ tales que $:|\lambda| \leqslant 1,|\mu| \leqslant 1$ se tiene $\lambda x+\mu y \varepsilon S$.

$\mu=0)$

Nötese que un K-convexo es equilibrado (tomando:

Teorema 1 :

Si $S \in E, S K$-convexo; entonces $S=\stackrel{0}{S} \sigma \quad \stackrel{0}{S}=\emptyset$

DM :

$$
\begin{aligned}
& \text { Si } \stackrel{\circ}{S} \neq \varnothing \\
& \text { Sean : }\left\{\begin{array}{l}
x \in S \\
U \text { vecindad de } 0 \text { tal que } x+U<S
\end{array}\right. \\
& \text { si : y } \varepsilon \text { s; entonces: } y-x=y+(-1) x \varepsilon s \\
& \text { además : } \forall u \in U, x+4 \varepsilon S \\
& \text { Iuego: } y+u=(y-x)+(x+u) \varepsilon S \text {, } u \varepsilon U \\
& y+u<s \\
& \text { Ejemplo en que } S=\emptyset:
\end{aligned}
$$$$
\text { sea } K n \cdot a \cdot y \text { sea } E=\left\{\left(\alpha_{n}\right) / \alpha_{n} \varepsilon k, \alpha_{n}=0\right.
$$

salvo para finitos $n$ \}

sobre E consideramos la topología inducida por la norma:

topológico.

$$
\left(\alpha_{i}\right) \rightarrow\left\|\left(\alpha_{i}\right)\right\|=\max \left|\alpha_{i}\right|
$$

Entonces (E, \|\|$)$ es un espacio vectorial

$$
\text { Sea } S=\left\{\left(\alpha_{i}\right) \varepsilon E /\left|\alpha_{i}\right|<\rho^{-i}, \forall i \varepsilon N\right\} \text {, donde }
$$

$\rho>1$, real, tal que: $V n \varepsilon Z /, \lambda_{n} \in K$ tal que: 
$\left|\lambda_{1}\right|=\rho^{n} \cdot(\rho$ existe, si $\circ \varepsilon k$ tal que $|\alpha|=0>1$ ) Consideremos la sucesjón $\left(x_{n}\right)$ tal que:

$$
\begin{aligned}
& x_{n}=\left(\alpha_{n i}\right) \text {, donde : } \\
& \alpha_{n i}= \begin{cases}0 & \text { si } i \neq r \\
\rho^{-n} \text { si } i=r\end{cases}
\end{aligned}
$$

Asi $|x n|=\rho^{-n}, \forall n \varepsilon N$

de donde $:(x n) \rightarrow 0$

Pero, $\forall n \varepsilon N, x_{n} \varepsilon S$; lo que significa que hav una vecindad de 0 que no esta contenida en $S$.

$$
\begin{aligned}
& \therefore \quad 0 \text { no es punto interior de } S \\
& \therefore \quad S=\emptyset
\end{aligned}
$$

Es sencillo comprobar que la intersección de una familia arbitraria de $K$-convexos es un K-convexo. Se defi ne entonces la cápsula K-convexa de $S, C(s)$ como el menor $K$-convexo que contiene a $S$.

Teorema 2:

$$
\text { Si s es abierto: } C(S) \text { es abierto. }
$$

$\underline{D M}$.

$$
\begin{aligned}
& S \text { vecindad abierta no vacia. } \\
& S \leq c(S) \Rightarrow c(S) \neq \emptyset \\
& \text { por teorema } 1: c(S)=c(S)
\end{aligned}
$$

Una topologia compatible con la estructura Iineal de $E$ se llama localmente K-convexa, si tiene un filtro de vecindades de 0 formado por conjuntos $K$-convexos. En ese ca so E se llama localmente K-convexo. 
Se llama semi-norma no arquimediana sobre $E$ a aplicación $p: E \rightarrow R$ que satisface:

$$
\begin{aligned}
& S N \quad \text { 1) } p(\lambda x)=|\lambda| p(x) \\
& S N \quad \text { 2) } p(x+y) \leqslant \operatorname{mix}\{p(x), p(y)\}
\end{aligned}
$$

Teorema 3 :

Sea $S C E, \quad K$-convexi y absorbente y $p_{S}: E \rightarrow F$ la aplicación definida por :

$$
P_{S}(x)=\inf _{x \in \lambda S}|\lambda|
$$

\section{Entonces :}

nua.

1) $P_{s}$ es una s.n.n.a. sobre $E$

2) Si s contiene un punto interior $\mathrm{P}_{\mathrm{S}}$ es conti

$\underline{\mathrm{DM}}$.

1) $\operatorname{SN~1):~} \alpha x \varepsilon \lambda S \leftrightarrow x \varepsilon \lambda \alpha^{-1} S$

asi:

$$
p_{S}(\alpha x)=\inf \{|\lambda| / \alpha x \varepsilon \lambda S\}
$$

$=\inf \left\{|\lambda| / x \varepsilon \alpha^{-1} \lambda s\right\}$ : haciendo $\beta=\alpha^{-1} \lambda$

$=\inf \{|\alpha \beta| / x \in \beta \quad S\}$

$=|\alpha| \inf \left\{\left\{\beta \mid / x \in x_{S}(x)\right.\right.$

SN 2) Sean $\lambda_{0}, \lambda_{1} \in \quad K$ puntos que :

$$
\mathrm{x} \quad \varepsilon \quad \lambda_{0} \mathrm{~S}, \mathrm{y} \quad \varepsilon \quad \lambda_{1} \mathrm{~S}
$$

entonces: si $\left|\lambda_{2}\right|=\max \left\{\left|\lambda_{1}\right|\left|\lambda_{0}\right|\right\}$

se tiene: $\lambda_{0} \lambda_{2}^{-1} s_{1}+1 \lambda_{2}^{-1} s_{2} \varepsilon s, \forall s_{1}, s_{2} \varepsilon s$

o sea : $\lambda_{0} \lambda_{2}^{-1} \mathrm{~s}+\lambda_{1} i_{2}^{-1} \mathrm{~s}<\mathrm{s}$

Luego: $\lambda_{0} S+\lambda_{1} S<\lambda_{2} S$ 


$$
\begin{aligned}
& \text { en consecuencia: } \mathrm{x}+\mathrm{y} \varepsilon \lambda_{2} \mathrm{~s} \\
& \therefore \mathrm{P}_{\mathrm{S}}(\mathrm{x}+\mathrm{y})<\left|\lambda_{2}\right|=\max \quad\left\{\left|\lambda_{1}\right|,\left|\lambda_{2}\right|\right\}
\end{aligned}
$$

2) Es consecuencia inmediata de 1) y Teorema 1).

III. TEOREMA DE HAHN - BANACH.

Ingleton, A.W, en "The Hahn-Banach Theonem for non - Archimedian valued fields" (Pric. Cambridge Phil. Society 48, 41 - 45); ha demostrado que: si E espacio vecto rial normado no arquimediano y si K es esférico - completo; entonces para una aplicación lineal continua de un sub-espa cio vectorial $M$ de $E$ en $K$, una aplicación lineal continua $\mathrm{T}^{*}$ de $\mathrm{E}$ en $K$, que satisface las condiciones siguientes:

$$
\begin{aligned}
& \text { 1) } \mathrm{T}^{*}(\mathrm{x})=\mathrm{T}(\mathrm{x}) \quad \forall \mathrm{x} \in \mathrm{E} \\
& \text { 2) } \sup _{\mathrm{x} \in M, \mathrm{x} \neq 0} \mathrm{~T}(\mathrm{x})\|/\| \mathrm{x}\left\|=\sup _{\mathrm{x} \in \mathrm{E}, \mathrm{x} \neq 0}\right\| \mathrm{T}^{*}(\mathrm{x})\|/\| \mathrm{x} \|
\end{aligned}
$$

Este resultado es la base del Teorema de Hahn Banach que se expone más adelante. Previamente veamos el siguiente teorema :

Teorema 1 :

Sean E y $F$ espacios localmente K-convexos $y$ $T: E \rightarrow F$ una aplicación Iineal. Para que $T$ sea continua es necesario y suficiente que para toda semi-norma n.a. con tinua $q$ sobre $F$, exista una semi-norma n.a. continua $p$. sobre $E$ y $\quad C \varepsilon R, c>0$ tal que:

$$
q(T(x)) \leqslant c \quad p(x), \forall x \in E \text {. }
$$

$\underline{D M}$

La suficiencia dela condición es evidente. Para demostrar la necesidad, sea q una $s-n$. n.a. y continua sobre F. Entonces existe una $s-n$. n.a. y continua p sobre $E$ tal que: 
$\forall x \in E, p(x) \leqslant 1$ implica $q(T(x)) \leqslant 1$

piedad :

sea $\rho<1$, tal que $\forall n \varepsilon z, \exists \lambda_{n} \varepsilon K$ con la pro-

$$
\left|\lambda_{n}\right|=o^{n} ; \text { si } p(x)=0 \text {, }
$$

entonces

$p\left(\lambda_{n} x\right)=0 \quad \forall n \varepsilon z$,

luego

de:

$q\left(T\left(\lambda_{n} x\right)\right)-\rho^{n} q(T(x)) \leqslant 1$, $\forall n \varepsilon Z$, de don $q(T))=0$

Si $p(x) \neq 0, \exists n \varepsilon z$ tal que :

$\rho^{n}<(p(x))^{-1} \leqslant \rho^{n+1}$

Entonces : $p\left(\lambda_{n} x\right)=\rho^{n} p(x)<1$, luego

$a(T(\lambda x))=\rho^{n} q(T(x) \leqslant 1$, de donde:

$q(T(x))=\rho^{n} q(T(x)) 0^{-r} \leqslant \rho p(x)$

Suponiendo que $E$ es localmente $K$-convexo y que $K$ es esfêrico-completo. En este caso, se tiene el siguiente Teorema :

Teorema 2 : (Hanhn-Banch).

Sean: M subespacio de E, p. un $s-n \cdot n \cdot a$. sobre $E$, $c>0$ en $R$ y $f: M \rightarrow K$ lineal tal que: $|f(x)| \leqslant c p(x)$ $\mathrm{V} \times \varepsilon \mathrm{M}$

Entonces: $f^{*}: E \rightarrow K$ lineal, que satisface:

1) $f^{*}(x)=f(x) \quad V x \in M$

2) $\left|f^{*}(x)\right| \leqslant c p(x) \quad V x \in E$

$\underline{D M}:$

Sea $L=\{x \in E / p(x)=0\}_{y} x \rightarrow \hat{x}$ la aplicación canô $\hat{E}=E / L$

Sea $\hat{M}$ la imagen de $M$ en $\hat{E}$. 

La aplicación $\hat{x} \rightarrow \mid \hat{x} \|=p(x)$ es una norma n. a
sobre $\hat{E}$.

Como $x \in M, p(x)=0 \rightarrow f(x)=0$, se puede definir una aplicación lineal $\hat{f}$ de $\hat{M}$ en K mediante la fórmula:

$\hat{f}(\hat{x})=f(x), \forall x \quad M$.

Se tiene que: $|\hat{f}(\hat{x})|=|f(x)| \leqslant c p(x)=c\|\hat{x}\|, \forall x \in M$.

Luego del resultado de Ingleton, es posible afirmar la existencia de una aplicación lineal

$\hat{f}^{*}: \hat{E} \rightarrow k$ que satisface

1) $\hat{f}^{*}(\hat{x})=\hat{f}(\hat{x}) \quad \forall \hat{x} \varepsilon \hat{M}$

2) $\left|\hat{f}^{*}(\hat{x}) ! \leqslant c\right| \hat{x} \mid \quad \forall \hat{x} \varepsilon \hat{E}$

La aplicación de $f^{*}: E \rightarrow K$, definida por

$f^{*}(x)=\hat{f}^{*}(\hat{x})$ es la función buscada.

Teorema 3 :

Sea M subespacio vectorial de E y $f$ una aplica -
ción lineal continua de M en K. Entonces existe $f^{*}: E \rightarrow K$ tal que: $f^{*}(x)=f(x), \forall x \varepsilon M$

$\underline{\mathrm{DM}}:$

Por teorema 1., una semi-norma n.a. continua p sobre E $\mathrm{y} \quad \mathrm{c} \varepsilon \mathrm{R}, \quad \mathrm{c}>0$

tal que: $f(x) \leqslant c p(x), \forall x \in M$.

cación $f^{*}$ Por teor 